\title{
Effects of Supervised Versus Unsupervised Aerobic Exercise Training on Weight Loss, Functional Capacity, Quality of Life and Depression Level in Patients With Essential Hypertension
}

\author{
İlknur Albayrak ${ }^{1}$, İlyas Kaya ${ }^{2}$, Ayşe Güleç², Hasan Kara ${ }^{3}$, Elif Balevi Batur ${ }^{2}$, and Funda \\ Levendoğlu ${ }^{2}$ \\ ${ }^{1}$ Affiliation not available \\ ${ }^{2}$ Selçuk University Faculty of Medicine \\ ${ }^{3}$ Erzurum Horasan State Hospital, Department of Physical Medicine and Rehabilitation
}

February 19, 2021

\begin{abstract}
Purpose: This study aimed to evaluate the effects of a supervised aerobic exercise training program on weight loss, functional capacity, quality of life(QoL) and depression level in patients with essential hypertension. Methods: This is a prospective study. Patients who chose the treatment type according to personal preferences were divided into two groups: Group 1(n:91) exercised for six weeks, five sessions per week under the supervision of a medical doctor in the aerobic exercise laboratory and group 2(n:47) declared to perform a walking in the free time, at least twice a week, but without supervision. Body weight, serum lipid levels, functional capacity, QoL, and depression level of all the participants were evaluated at the pre-treatment period and the post-treatment 6 th week. Results: When the two groups were compared, a significant increase in the six-minute walk test(6MWT) distance and SF-36 subscales of physical function, role physical, physical component summary, vitality, role emotional and mental component summary in Group 1 at the post-treatment 6th week was found in Group 1 . There was a significant reduction in the resting systolic blood pressure(SBP), weight, body mass index and Beck Depression Inventory scores between pre-treatment and post-treatment 6th week in Group 1. There was a significant increase in the METs, 6MWT, SF36 subscales of physical function, role physical, physical component summary, vitality, role emotional and mental component summary scores between pre-treatment and post-treatment 6th week in Group 1. Conclusion: Supervised aerobic exercise training of 6 weeks duration is more effective than unsupervised aerobic exercise training for the functional capacity and QoL in patients with hypertension. Supervised aerobic exercise as antihypertensive lifestyle therapy should be recommended for patient compliance, absence of adverse incidents, and its positive impact on resting SBP, weight, functional capacity, QoL, and depression level in patients with hypertension.
\end{abstract}

Effects of Supervised Versus Unsupervised Aerobic Exercise Training on Weight Loss, Functional Capacity, Quality of Life and Depression Level in Patients With Essential Hypertension

\section{Abstract}

Purpose: This study aimed to evaluate the effects of a supervised aerobic exercise training program on weight loss, functional capacity, quality of life(QoL) and depression level in patients with essential hypertension.

Methods: This is a prospective study. Patients who chose the treatment type according to personal preferences were divided into two groups: Group 1(n:91) exercised for six weeks, five sessions per week under the supervision of a medical doctor in the aerobic exercise laboratory and group 2(n:47) declared to perform 
a walking in the free time, at least twice a week, but without supervision. Body weight, serum lipid levels, functional capacity, QoL, and depression level of all the participants were evaluated at the pre-treatment period and the post-treatment 6 th week.

Results: When the two groups were compared, a significant increase in the six-minute walk test(6MWT) distance and SF-36 subscales of physical function, role physical, physical component summary, vitality, role emotional and mental component summary in Group 1 at the post-treatment 6th week was found in Group 1. There was a significant reduction in the resting systolic blood pressure(SBP), weight, body mass index and Beck Depression Inventory scores between pre-treatment and post-treatment 6th week in Group 1. There was a significant increase in the METs, 6MWT, SF-36 subscales of physical function, role physical, physical component summary, vitality, role emotional and mental component summary scores between pre-treatment and post-treatment 6th week in Group 1.

Conclusion: Supervised aerobic exercise training of 6 weeks duration is more effective than unsupervised aerobic exercise training for the functional capacity and QoL in patients with hypertension. Supervised aerobic exercise as antihypertensive lifestyle therapy should be recommended for patient compliance, absence of adverse incidents, and its positive impact on resting SBP, weight, functional capacity, QoL, and depression level in patients with hypertension.

Key words: supervised aerobic exercise, weight loss, functional capacity, quality of life, depression level, essential hypertension

\section{What is already known about this topic?}

The regular exercise, mainly by performing aerobic exercise, is a critical modifiable behavioral determinant of hypertension and is recommended as a complementary therapy for the prevention and treatment of hypertension. ${ }^{1}$ Previous studies have reported that hypertension patients have low long-term compliance with exercise programs. ${ }^{3,13}$ Aerobic exercise training relates to cardiovascular health such as functional capacity, weight loss, QoL and depression level in patients with essential hypertension. ${ }^{14-22}$ Although it is important, the effect of supervised versus unsupervised aerobic exercise training program on weight loss, functional capacity, QoL and depression level in hypertensive subjects has been poorly investigated ${ }^{13,17,18,23}$ and none of the studies has examined all these parameters collectively in the same patient group. ${ }^{24,25}$

\section{What does this article add?}

Results of this study add to the literature that the supervised aerobic exercise training of 6 weeks duration is more effective than unsupervised aerobic exercise training for the functional capacity and QoL in patients with hypertension. These findings may have important implications in the design of exercise programs for the hypertensive patients. Supervised aerobic exercise as antihypertensive lifestyle therapy should be recommended for patient compliance, absence of adverse incidents, and its positive impact on resting SBP, weight, functional capacity, QoL, and depression level in patients with hypertension.

\section{Introduction}

Hypertension is the most common and modifiable cardiovascular disease (CVD) risk factor, due to stroke, myocardial infarction and sudden death. ${ }^{1}$ Hypertension is estimated to affect 1.39 billion adults $(31 \%)$ worldwide $^{2}$ and is being responsible for 8 million deaths per year. ${ }^{3}$

Low physical activity is a strong predictor for future cardiovascular morbidity and mortality in patients with hypertension. ${ }^{4,5}$ Many researchers have shown that a single episode of exercise can reduce blood pressure (BP), and physically active individuals have a lower risk of becoming hypertensive than do sedentary individuals. ${ }^{6,7}$ Physical activity has a positive effect on hypertension by activating the sympathetic nervous system, endothelial function, insulin sensitivity, and the renin-angiotensin system. ${ }^{8,9}$ The regular exercise, mainly by performing aerobic exercise, is a critical modifiable behavioral determinant of hypertension and is recommended as a complementary therapy for the prevention and treatment of hypertension. ${ }^{1}$ 
The meta-analysis of 26 randomized controlled studies (RCS) have concluded that, aerobic exercise is effective in reducing clinical $\mathrm{BP}$ in hypertensive subjects $(-8.3 \mathrm{mmHg}$, systolic blood pressure (SBP) and $-5.2 \mathrm{mmHg}$ diastolic blood pressure (DBP)) with training sessions conducted 2-3 times per week, lasting 30-45 min and with moderate intensity. ${ }^{10}$ Therefore, aerobic exercise is recommended by current American and European hypertension guidelines to reduce BP. ${ }^{11,12}$

Previous studies have reported that hypertension patients have low long-term compliance with exercise programs. ${ }^{3,13}$ The poor adherence to exercise as antihypertensive therapy underscores the need for the supervised aerobic exercise training to increase the motivation to exercise that possibly would favorably impact exercise adherence among patients with hypertension. ${ }^{3}$

Aerobic exercise training relates to cardiovascular health such as functional capacity, weight loss, quality of life (QoL) and depression level in patients with hypertension. A significant factor in the effectiveness of aerobic exercise is their positive effect on patients' functional capacity, which results in improved prognosis and survival after diagnosis of hypertension. ${ }^{14}$ Functional capacity following a cardiovascular event is known to be a strong independent predictor of mortality. ${ }^{15}$ Aerobic training includes interventions specifically addressing to weight loss, such as high caloric energy expenditure exercise training. ${ }^{16,17} \mathrm{~A}$ few studies examined the effect of aerobic exercise training on weight and QoL have shown that decreases in body mass index (BMI) also came with significant improvements in QoL. ${ }^{17,18}$ According to a meta-analysis of 33 studies, QoL of hypertensive patients is worse than that of normotensive individuals. ${ }^{19}$ Previous studies indicate that psychological distress is a significant risk factor for CVD, such as hypertension and adversely affects recovery following hypertension. ${ }^{17,20}$ Many studies have shown that aerobic exercise has potentially positive effects on some psychiatric conditions, such as anxiety and depression. ${ }^{18,21,22}$

Although it is important, the effect of supervised versus unsupervised aerobic exercise training program on weight loss, functional capacity, QoL and depression level in hypertensive subjects has been poorly investigated $^{13,17,18,23}$ and none of the studies has examined all these parameters collectively in the same patient group. ${ }^{24,25}$ Thus, the present trial aimed to evaluate the effects of a supervised aerobic exercise training program on weight loss, functional capacity, QoL and depression level in patients with essential hypertension. We hypothesized that the supervised aerobic exercise program would improve functional capacity and QoL, reduce weight and depression level, compared to an unsupervised aerobic exercise program.

\section{Methods}

\section{Study population}

This study was conducted at the Department of Physical Medicine and Rehabilitation between November 2017 and April 2019. A total of 155 patients were selected who had controlled arterial hypertension diagnosed more than one year earlier and had taken the same antihypertensive medications for at least three months. The inclusion criteria were: over 18 years of age, absence of concomitant other metabolic and cardiovascular diseases. Exclusion criteria were: having suffered an event of ischaemic heart disease $(<6$ months), secondary hypertension, hypertrophic obstructive cardiomyopathy, congestive heart failure, uncontrolled cardiac arrhythmia, symptomatic peripheral arterial occlusive disease, aortic insufficiency or stenosis, pulmonary or heart disease with dyspnoea at small or moderate effort, an outbreak of orthopedic problems on hip, knee or ankles, such as arthroplasty, contracture or severe osteoarthritis, using of antidepressant or anxiolytic drugs, pregnancy, cognitive disfunction. Patients signed an informed consent form before being included in the study. The study was approved by the local ethics committee of the Selcuk University Medical Faculty.

\section{Procedure and intervention}

Participants were followed for routine management of diet, weight and BP. We did not make any antihypertensive medication changes during the study period of 6 weeks. There was no randomization in the type of treatments or in the patients to be chosen. All patients chose the treatment type according to personal preferences. Patients who chose the treatment type according to personal preferences were divided into two 
groups:

1. Group 1 exercised for six weeks, involving once per day, five sessions per week under the supervision of a medical doctor, and a nurse in the aerobic exercise laboratory. The aerobic exercise training program lasted six weeks, and patients lived in the hospital for the whole period. Aerobic exercise training was performed on cycle ergometers (Ergoline, ergoselect II 100/200/Reha, Germany) equipped with a computed ergometer and developed to monitor electrocardiography (ECG), heart rhythm and BP. Each session consisted of a 5-minute warm-up, followed by 50 minutes of aerobic exercise with an intensity of $50 \%$ to $70 \%$ of heart rate reserve, calculated by Karvonen formula, and ended with a 5 -min cool-down period. ${ }^{26,27}$

2. Group 2 received a standard recommendation for patients with hypertension, having declared to perform a walking in the free time, at least twice a week over the experimental period, but without supervision. ${ }^{13}$

\section{Blood pressure}

After a ten minutes resting in a supine position, office BP was measured from the brachial artery using a mercury sphygmomanometer (ERKA D-83646 Bad Tolz, Kallmeyer Medizintechnik GmbP Co. KG, Germany).

\section{Exercise stress test}

All patients in group 1 were performed the exercise test on a treadmill following the Bruce Protocol with continuous 12-lead electrocardiograph recording (Cambridge Heart, Inc. Exercise System CH 2000, GB) before initiating the aerobic training program. ${ }^{28}$ For each patient, test readings were recorded with 12 derivated ECG at the beginning, every 3 minutes during the test and at the 2nd minute of resting period.

\section{Outcome measures}

A detailed history and physical examination were obtained while including the patients into the study. All the participants completed the questionnaire, which gathered demographic data (age, gender, marital status, education level and occupation). Baseline body weight (using calibrated electronic digital scales [Oncomed; SC-105, California, America]) was measured at the pre-treatment period and the post-treatment 6th week for all the participants by the same physician.

Serum total cholesterol, high-density lipoprotein (HDL) cholesterol, low-density lipoprotein (LDL) cholesterol, very-low-density lipoprotein (VLDL) cholesterol and triglycerides were measured with the use of standard enzymatic kits at the pre-treatment period and the post-treatment 6 th week for all the participants. Twelve-hour fasting blood samples were collected between $6.00 \mathrm{am}$, and $10.00 \mathrm{am}$ at baseline and week 6 .

The functional capacity, QoL, and depression level of all the participants were evaluated by the six-minute walk test (6MWT), Short Form-36 (SF-36) and Beck Depression Inventory (BDI), respectively from faceto-face interviews at the pre-treatment period and the post-treatment 6 th week. The estimated exercise capacity (estimated metabolic equivalents or METs) was assessed at baseline and week 6 . These instruments were recorded by the same physician who was blinded to the type of treatment for each patient.

\section{Assessment of functional capacity}

The 6MWT test and the estimated metabolic equivalents or METs were used performed to evaluate functional capacity. The 6MWTs were performed along a flat, 30-m long hospital corridor, with marks at $3 \mathrm{~m}$ intervals for all the participants. Each $6 \mathrm{MWT}$ was supervised by a medical doctor. ${ }^{9}$ The estimated METs were assessed for all patients in group 1.

\section{Assessment of QoL}

SF-36, which was used to evaluate the QoL, consists of 36 questions and two components. The physical component summary comprises four domains (physical function, role physical, bodily pain and general health) and the other four the mental component summary (vitality, role emotional, social function, and mental health). Scores range from 0 to 100 , with high scores indicating a better QoL. ${ }^{29}$ 


\section{Assessment of depression level}

BDI was used to assess the depression level of the participants. BDI is a 21-item self-report questionnaire. The questionnaire has four statements. These statements are graded from 0 to $3{ }^{30}$ The score higher than 17 denotes the presence of depression.

\section{Statistical analyses}

Kolmogorov-Smirnov normality tests were conducted for all continuous data. Comparisons between preand post-treatment values were evaluated using Student's dependent samples t-test. Nonparametric MannWhitney or Chi-square tests $\left(\mathrm{x}^{2}\right)$ were performed to compare socio-demographic and clinical measures between the two groups. All categorical variables were described as percentages, while means and standard deviations were reported for continuous variables. The level of significance was set at $\mathrm{p}<0.05$.

\section{Results}

None of the patients was given any treatment other than that described earlier, and no complications were observed in the patients. The study was completed with a total of 138 patients (Figure 1). There were 91 patients in the group 1 and 47 patients in the group 2. There were no statistically significant differences between the two groups in terms of age, sex, marital status, educational level and occupation. Their demographic and clinical characteristics are shown in Table 1.

\section{Between-groups}

There was a significant difference in total cholesterol, LDL levels, and role physical score during pre-treatment between the two groups $(\mathrm{p}=0.01, \mathrm{p}=0.002, \mathrm{p}=0.007$, respectively). A comparison of resting $\mathrm{SBP}$, resting DBP, resting heart rate, weight, body mass index (BMI), total cholesterol, HDL, LDL, VLDL, triglycerides, METs, 6MWT scores between the two groups for the pre-treatment period and the post-treatment 6th week was shown in Table 2. When the two groups were compared, a significant increase in 6MWT distance and SF-36 subscales of physical function, role physical, physical component summary, vitality, role emotional and mental component summary in Group 1 at the post-treatment 6th week was found in Group $1(\mathrm{p}<0.05)$.

\section{Within-groups}

Resting SBP, resting DBP, resting heart rate, weight, BMI, total cholesterol, HDL, LDL, VLDL, triglycerides, METs, 6MWT scores between pre-treatment and post-treatment 6th week in both groups are shown in Tables 2.

There was a significant reduction in the resting SBP, weight, BMI and BDI scores between pre-treatment and post-treatment 6th week in Group $1(\mathrm{p}<0.05)$. There was a significant increase in the METs, 6MWT, SF-36 subscales of physical function, role physical, physical component summary, vitality, role emotional and mental component summary scores between pre-treatment and post-treatment 6th week in Group 1(p<0.05).

\section{Discussion}

This study has shown that the supervised aerobic exercise training of 6 weeks duration can reduce resting SBP, weight, depression level and to increase functional capacity and QoL in patients with essential hypertension. The supervised aerobic exercise training is more effective than unsupervised aerobic exercise training for the functional capacity and QoL in patients with essential hypertension. Collectively, our findings indicated that the supervised aerobic exercise is superior to unsupervised aerobic exercise as antihypertensive therapy and should recommended for its benefits.

Aerobic exercise is a cornerstone of the lifestyle recommendations in current hypertension guidelines. ${ }^{31}$ The meta-analysis of $28 \mathrm{RCS}$ have concluded a mean reduction of $\mathrm{BP}$ of $12 \mathrm{mmHg} \mathrm{SBP}$, thus supporting the antihypertensive effects of aerobic exercise. ${ }^{32}$ Tabara and associates ${ }^{33}$ found that aerobic exercise could lower resting SBP by 5 to $15 \mathrm{mmHg}$, and resting DBP by 4 to $9 \mathrm{mmHg}$ in patients with essential hypertension. In the present study, we found that the supervised aerobic exercise training of 6 weeks duration reduced resting SBP by $4.4 \pm 12.8 \mathrm{mmHg}$ and resting DBP $2 \pm 12.8 \mathrm{mmHg}$ in patients with hypertension. There was no 
reduction in resting $\mathrm{BP}$ in the unsupervised aerobic exercise group. This result shows that the unsupervised aerobic exercise group had poor adherence. If aerobic exercise is a cardiovascular pill, adherence to this ideal 'drug' is essential. Therefore, supervised aerobic exercise training is crucial in terms of compliance and beneficial effects.

Obesity is predisposed to CVD by increasing blood pressure, increasing plasma lipid levels, and causing sedentary life. ${ }^{17}$ According to the previous studies, obese patients may acquire significant health benefits from improvement in physical activity level and change in weight. ${ }^{17,34}$ Weight reduction with aerobic exercise training has significant effects on increasing levels of functional capacity, QoL, and psychological health. ${ }^{17,18}$ There is strong evidence in the general population that physical activity is a determining factor in the decrease and maintenance of a healthy weight. ${ }^{18}$ A RCT with a physical activity program of $165-220 \mathrm{~min} /$ week with hypertensive patients reported a significant reduction in body weight $(-1.8 \mathrm{~kg})$ and BMI $\left(-0.6 \mathrm{~kg} / \mathrm{m}^{2}\right)$, results for the present study $\left(-3.2 \mathrm{~kg}\right.$ and $-1.2 \mathrm{~kg} / \mathrm{m}^{2}$, respectively) were better than in that study. ${ }^{34}$ In this study, while weight reduction in supervised aerobic exercise training group was $3.6 \%$, there was a $0.6 \%$ reduction in body weight in unsupervised aerobic exercise training group during 6 weeks. According to the results of this study, we consider that supervised aerobic exercise training as a physical activity is more successful in weight loss in patients with hypertension.

Exercise training has also been shown to reduce triglycerides and LDL cholesterol, as well as increase in HDL cholesterol. ${ }^{35}$ It has not yet been established how much aerobic exercise is required in order to improve the lipid levels. ${ }^{36}$ According to the previous studies, the duration of aerobic exercise for improvement of serum lipid levels is mostly 8 weeks and over. ${ }^{13,37}$ In the present study, we found that supervised aerobic exercise training increased HDL cholesterol by $0.6 \mathrm{mg} / \mathrm{dl}$, and reduced LDL cholesterol and triglycerides by $1.3 \mathrm{mg} / \mathrm{dl}$ and $12.5 \mathrm{mg} / \mathrm{dl}$, respectively after 6 weeks. However, these differences were not statistically significant. According to the results of this study, if aerobic exercise duration is longer than 6 weeks, lipid levels can be more positively affected.

Previous studies show that improvement in functional capacity is considered an important predictive marker of survival in cardiovascular disease. ${ }^{15,17}$ In a study of 5721 asymptomatic women, for every increase in the functional capacity of $1 \mathrm{MET}$, the risk of death was reduced by $17 \% .^{38}$ Our results show that estimated METs improved by $0.8 \pm 1.1(26,7 \%)$ in supervised aerobic exercise training group, between baseline and the end of the intervention. Using the 6MWT, there was an increase of 48 meters (12.8\%) in supervised group at the end of 6 weeks, while there was an increase of 10 meters (2.7\%) in the unsupervised group. According to these results, the functional capacity improved approximately five times in the supervised aerobic exercise training group compared to the unsupervised group for hypertensive patients. In view of the considerable impact that the increase in functional capacity has on the health of hypertensive patients, these patients should be recommended a supervised aerobic exercise training.

Two RCTs with hypertensive patients reported that there is a positive effect of physical activity on QoL. ${ }^{24,25}$ Specific different designs of physical activity programs aimed at improving QoL and adherence to exercise have been reported, such as counseling, supervision and group interventions. ${ }^{18}$ Therefore, supervised and unsupervised aerobic exercise programs were compared in the present study. When the two groups were compared, the benefits of the supervised aerobic exercise training on QoL were observed in some domains such as physical function, role physical, physical component summary, vitality, role emotional, and mental component summary after 6 weeks for hypertensive patients. The explanation may be of increased functional capacity, weight loss, and adherence to exercise in the supervised aerobic exercise training group. It seems that the supervised aerobic exercise training, such as the present study, have had favourable effects on the QoL for hypertensive patients.

Previous studies show that psychological distress play an essential role in the pathogenesis and prognosis for CVD. ${ }^{17,20}$ Physical activity, such as aerobic exercise, has potentially beneficial effects on psychological status. ${ }^{18,21}$ The sense of well-being associated with the aerobic exercise training may be related to the release of neurotransmitters, which act at the brain level, increasing the feeling of well-being. ${ }^{18}$ Weight loss from aerobic exercise training may contribute to reducing depression levels. ${ }^{17}$ In this study, during 6 weeks, while 
the reduction of BDI score in supervised aerobic exercise training group was $4.2 \pm 5.2$, there was $0.4 \pm 1.9$ in the reduction of BDI score in unsupervised aerobic exercise training group in patients with hypertension. Although there was no significant difference between the two groups in terms of depression level, a noticeable improvement was observed in the supervised aerobic exercise group compared to pre-treatment. These results show that if proper exercise compliance was provided, the depression level was reduced for hypertensive patients.

Several potentially important study limitations deserve attention. First, a control group was not included. There is an ethical obligation to guarantee adequate treatment to every patient for the control group. ${ }^{17}$ Second, there was no randomization in the type of treatments or in the patients to be chosen. All patients were allocated in the experimental groups according to their desire to participate in the supervised or unsupervised exercise program. Third, the present study has no long term follow-up assessments, making thus impossible to know if observed short-term improvements were maintained in the long-term. Finally, exercise frequency, duration, intensity, and sessions were not precise for the unsupervised aerobic exercise training group. These patients were recommended to perform a walking in their free time, at least twice a week, over the experimental period. Randomized studies, including a control group and long term follow-up assessments might better show the effectiveness of supervised versus unsupervised aerobic exercise training in patients with hypertension.

In summary, results of this study add to the literature that the supervised aerobic exercise training of 6 weeks duration is more effective than unsupervised aerobic exercise training for the functional capacity and QoL in patients with hypertension. These findings may have important implications in the design of exercise programs for the hypertensive patients. Supervised aerobic exercise as antihypertensive lifestyle therapy should be recommended for patient compliance, absence of adverse incidents, and its positive impact on resting SBP, weight, functional capacity, QoL, and depression level in patients with hypertension.

Disclosure: İlknur Albayrak declares that she has no conflict of interest concerning the research, authorship or publication of this article. Ilyas Kaya declares that he has no conflict of interest concerning the research, authorship or publication of this article. Ayşe Güleç declares that she has no conflict of interest concerning the research, authorship or publication of this article. Hasan Kara declares that he has no conflict of interest concerning the research, authorship or publication of this article. Elif Balevi Batur declares that she has no conflict of interest concerning the research, authorship or publication of this article. Funda Levendoğlu declares that she has no conflict of interest concerning the research, authorship or publication of this article.

Ethical approval: All procedures performed in studies involving human participants were in accordance with the ethical standards of the institutional and/or national research committee and with the 1964 Helsinki declaration and its later amendments or comparable ethical standards.

Funding: This research did not receive any specific grant from funding agencies in the public, commercial, or not-for-profit sectors.

Author contributions: İknur Albayrak: Conceptualization, Methodology, Software, Validation, WritingOriginal draft preparation, Supervision Illyas Kaya: Resources, Data curation, Software Ayşe Güleç: Resources, Data curation Hasan Kara: Resources Elif Balevi Batur: Writing - Review \& Editing, Visualization Funda Levendoğlu: Writing- Reviewing and Editing.

\section{References}

1. Brito LC, Peçanha T, Fecchio RY, et al. Morning versus evening aerobic training effects on blood pressure in treated hypertension.Med Sci Sports Exerc. 2019;51:653-662.

2. Mills KT, Bundy JD, Kelly TN, et al. Global disparities of hypertension prevalence and control: a systematic analysis of population-based studies from 90 countries. Circulation2016;134:441-450.

3. Zaleskia AL, Taylora BA, Park CL, et al. Using the immediate blood pressure benefits of exercise to improve exercise adherence among adults with hypertension: a randomized clinical trial. J Hypertens.2019;37:1877- 
1888.

4. Ratnaparkhe V, Bhangale A. Left ventricular diastolic dysfunction in primary hypertension and its relation with leisure time physical activity. J Assoc Physicians India. 2015;63:20-24.

5. Zeno SA, Kim-Dorner SJ, Deuster PA, Davis JL, Remaley AT, Poth M. Cardiovascular fitness and risk factors of healthy African Americans and Caucasians. J Natl Med Assoc. 2010;102:28-35.

6. Cardoso CG Jr, Gomides RS, Queiroz AC, et al. Acute and chronic effects of aerobic and resistance exercise on ambulatory blood pressure. Clinics (Sao Paulo). 2010;65:317-325.

7. Fagard RH. Exercise is good for your blood pressure: effects of endurance training and resistance training. Clin Exp Pharmacol Physiol. 2006;33:853-856.

8. Baghaiee B, Karimi P, Ebrahimi K, et al. Effects of a 12-week aerobic exercise on markers of hypertension in men. J Cardiovasc Thorac Res. 2018;10:162-168.

9. He LI, Wei WR, Can Z. Effects of 12-week brisk walking training on exercise blood pressure in elderly patients with essential hypertension: a pilot study. Clin Exp Hypertens . 2018;40:673-679.

10. Cornelissen VA, Smart NA. Exercise training for blood pressure: a systematic review and meta-analysis. $J$ Am Heart Assoc.2013;2:e004473.

11. Mancia G, Fagard R, Narkiewicz K, et al. 2013 ESH/ESC practice guidelines for the management of arterial hypertension. Blood Press. 2014;23:3-16.

12. James PA, Oparil S, Carter BL, et al. 2014 Evidence-based guideline for the management ofhigh blood pressure in adults: report from the panel members appointed to the Eighth Joint National Committee (JNC 8).JAMA. 2014;311:507-520.

13. Lima AM, Werneck AO, Cyrino E, Farinatti P. Supervised training in primary care units but not selfdirected physical activity lowered cardiovascular risk in Brazilian lowincome patients: a controlled trial. $B M C$ Public Health 2019;19:1738.

14. Branco C, Viamonte S, Matos C, et al. Predictors of changes in functional capacity on acardiac rehabilitation program. Rev Port Cardiol. 2016;35:215-224.

15. Jaureguizar KV, Vicente-Campos D, Bautista LR, et al. Effect of high-intensity interval versus continuous exercise training on functional capacity and quality of life in patients with coronary artery disease. A randomized clinical trial. J Cardiopulm Rehabil Prev.2016;36:96-105.

16. Ades PA, Savage PD, Toth MJ, et al. High-calorie-expenditure exercise: A new approach to cardiac rehabilitation for overweight coronary patients. Circulation 2009;119:2671-2678.

17.Manzoni GM, Villa V, Compare A, et al. Short-term effects of a multi-disciplinary cardiac rehabilitation programme on psychological well-being, exercise capacity and weight in a sample of obese in-patients with coronary heart disease: a practice-level study.Psychol Health Med. 2011;16:178-189.

18. Arija V, Villalobos F, Pedret R, et al. Physical activity, cardiovascular health, quality of life and blood pressure control in hypertensive subjects: randomized clinical trial. Health Qual Life Outcomes. 2018;16:184.

19. Ye R, Liu K, Zhang Z, Gong S, Chen X. Health-related quality of life of hypertension in China: a systematic review and meta-analysis. J Cardiovasc Med (Hagerstown). 2018;19:430-438.

20. Rozanski A, Blumenthal JA, Kaplan J. Impact of psychological factors on the pathogenesis of cardiovascular disease and implications for therapy. Circulation 1999;99:2192-2217.

21. Imboden C, Gerber M, Beck J, et al. Effects of aerobic exercise as add-on Treatment for inpatients with moderate to severe depression on depression severity, sleep, cognition, psychological well-being, and 
biomarkers: study protocol, description of study population, and manipulation check. Front Psychiatry. 2019;10:262.

22. Larun L, Brurberg KG, Odgaard-Jensen J, Price JR. Exercise therapy for chronic fatigue syndrome. Cochrane Database Syst Rev.2016;(6):CD003200.

23. Molmen-Hansen HE, Stolen T, Tjonna AE, et al. Aerobic interval training reduces blood pressure and improves myocardial function in hypertensive patients. Eur J Prev Cardiol. 2012;19:151-160.

24. Tsai J, Yang H, Wang W, et al. The beneficial effect of regular endurance exercise training on blood pressure and quality of life in patients with hypertension. Clin Exp Hypertens. 2004;26:255-265.

25. Sun J, Buys N. Community-based mind-body meditative tai chi program and its effects on improvement of blood pressure, weight, renal function, serum lipoprotein, and quality of life in Chinese adults with hypertension. Am J Cardiol. 2015;116:1076-1081.

26. Karvonen MJ, Kentala E, Mustala O. The effects of training on heart rate; a longitudinal study. Ann Med Exp Biol Fenn.1957;35:307-315.

27. Yaylalı YT, Fındıkoğlu G, Yurtdaş M, Konukçu S, Şenol H. The effects of baseline heart rate recovery normality and exercise training protocol on heart rate recovery in patients with heart failure. Anatol $J$ Cardiol. 2015;15:727-734.

28. Ash GI, Taylor BA, Thompson PD, et al. The antihypertensive effects of aerobic versus isometric handgrip resistance exercise. J Hypertens. 2017;35:291-299.

29. Albayrak I, Yilmaz H, Akkurt HE, Salli A, Karaca G. Is pain the only symptom in patients with benign joint hypermobility syndrome? Clin Rheumatol. 2015;34:1613-1619.

30. Beck AT, Guth D, Steer RA, Ball R. Screening for major depression disorders in medical inpatients with the Beck Depression Inventory for Primary Care. Behav Res Ther. 1997;35:785-791.

31. Pagonasa N, Vlatsasc S, Bauera F, et al. Aerobic versus isometric handgrip exercise in hypertension: a randomized controlled trial.J Hypertens. 2017;35:2199-2206.

32. Cornelissen VA, Fagard RH, Coeckelberghs E, Vanhees L. Impact of resistance training on blood pressure and other cardiovascular risk factors: a meta-analysis of randomized, controlled trials.Hypertension. 2011;58:950-958.

33. Tabara Y, Yuasa T, Oshiumi A, et al. Effect of acute and longterm aerobic exercise on arterialstiffness in the elderly. Hypertens Research: Official Journal JpnSoc Hypertens. 2007;30:895-902.

34. Blumenthal JA, Sherwood A, Gullette ECD, et al. Exercise and weight loss reduce blood pressure in men and women with mild hypertension. Arch Intern Med. 2000;160:1947.

35. Ciolac EG, Bocchi EA, Bortolotto LA, Carvalho VO, Greve JMD, Guimaraes GV. Effects of highintensity aerobic interval training vs. moderate exercise on hemodynamic, metabolic and neuro-humoral abnormalities of young normotensive women at high familial risk for hypertension. Hypertens Res. 2010;33:836843.

36. Mann S, Beedie C, Jimenez A. Differential effects of aerobic exercise, resistance training and combined exercise modalities on cholesterol and the lipid profile: review, synthesis and recommendations. Sports Med. 2014;44:211-221.

37. Fett C, Fett W, Marchini J. Circuit weight training vs jogging in metabolic risk factors of overweight/obese women. Arq Bras Cardiol. 2009;93:519-525.

38. Gulati M, Pandey DK, Arnsdorf MF, et al. Exercise capacity and the risk of death in women: the St. James women take heart Project. Circulation. 2003;108:1554-1559. 
Tables Table 1. Demographic characteristics of Group 1 and Group 2. Group 1 (n=91) Group 2 (n=47) P value Age (year

Table 2. Clinical and functional outcomes after treatments and changes from baseline values.

\begin{tabular}{llllll}
\hline Group 1 $(\mathrm{n}=91)$ & Group 1 $(\mathrm{n}=91)$ & Group 1 $(\mathrm{n}=91)$ & Group 1 $(\mathrm{n}=91)$ & Group 2 $(\mathrm{n}=47)$ & Group 2 $\left(\mathrm{n}=4^{\prime}\right.$ \\
\hline & Pre-treatment & Post-treatment & Change & Pre-treatment & Post-treatmen \\
Resting SBP $(\mathrm{mm} \mathrm{Hg})$ & $119.7 \pm 11.9$ & $115.2 \pm 6.7$ & $-4.4 \pm 12.8^{\mathrm{a}}$ & $116.3 \pm 5.2$ & $117 \pm 5$ \\
Resting DBP (mm Hg) & $74.1 \pm 11.6$ & $72 \pm 6.8$ & $-2 \pm 12.8$ & $71 \pm 2$ & $71.6 \pm 3$ \\
Resting heart rate (beats/min) & $78.5 \pm 9.8$ & $77.1 \pm 8.4$ & $-1.3 \pm 8.9$ & $76.5 \pm 7.8$ & $76.4 \pm 7$ \\
Weight & $87.3 \pm 13.9$ & $84.1 \pm 13$ & $-3.2 \pm 2.1^{\mathrm{c}}$ & $84.5 \pm 11.9$ & $84 \pm 11.6$ \\
BMI & $34.8 \pm 5.2$ & $33.5 \pm 4.9$ & $-1.2 \pm 0.8^{\mathrm{c}}$ & $32.9 \pm 4.8$ & $32.5 \pm 4.6$ \\
Total cholesterol (mg/dl) & $196.5 \pm 37.6$ & $192.3 \pm 44.5$ & $-4.1 \pm 39$ & $213.7 \pm 36.3$ & $210.8 \pm 37.5$ \\
HDL (mg/dl) & $44.5 \pm 9.8$ & $45.1 \pm 9.9$ & $0.6 \pm 6.6$ & $44.4 \pm 11.2$ & $43.4 \pm 9.8$ \\
LDL (mg/dl) & $118.8 \pm 29.6$ & $117.5 \pm 33.6$ & $-1.3 \pm 31.8$ & $135.7 \pm 33$ & $133,9 \pm 33.7$ \\
VLDL (mg/dl) & $34 \pm 18.8$ & $31.3 \pm 12.1$ & $-2.7 \pm 16.4$ & $33.4 \pm 15$ & $31.4 \pm 14.1$ \\
Triglycerides (mg/dl) & $168.8 \pm 95.8$ & $156.3 \pm 60.7$ & $-12.5 \pm 83.3$ & $162.7 \pm 68.8$ & $160.2 \pm 72.2$ \\
METs & $3 \pm 0.7$ & $3.8 \pm 1.4$ & $0.8 \pm 1.1^{\mathrm{c}}$ & & \\
6MWT (m) & $376.1 \pm 53.4$ & $424.9 \pm 76.3$ & $48.7 \pm 53.3^{\mathrm{c}, \mathrm{f}}$ & $365.2 \pm 57.1$ & $354.5 \pm 87.3$ \\
SF-36 & & & & \\
Physical function & $37.3 \pm 21.9$ & $53.4 \pm 22.1$ & $16 \pm 18.5^{\mathrm{c}, \mathrm{e}}$ & $40.3 \pm 21.9$ & $42.6 \pm 24.1$ \\
Role physical & $26.3 \pm 37.7$ & $57.6 \pm 44.5$ & $31.3 \pm 40.4^{\mathrm{c}, \mathrm{f}}$ & $9.5 \pm 26.8$ & $19.1 \pm 38.3$ \\
Bodily pain & $47.6 \pm 21.1$ & $45.9 \pm 19.1$ & $-1.7 \pm 24.4$ & $45.5 \pm 18.7$ & $41.4 \pm 13$ \\
General health & $52.5 \pm 15.3$ & $54.1 \pm 14.1$ & $1,5 \pm 11.8$ & $57.3 \pm 9$ & $56.4 \pm 10.1$ \\
Physical component summary & $40.9 \pm 15.4$ & $52.4 \pm 16.6$ & $11.4 \pm 12.2^{\mathrm{c}, \mathrm{f}}$ & $40.7 \pm 9$ & $42.3 \pm 11.5$ \\
Vitality & $46.7 \pm 14$ & $51.2 \pm 15.4$ & $4.5 \pm 13.3^{\mathrm{b}, \mathrm{d}}$ & $45.1 \pm 13.3$ & $45.2 \pm 12.4$ \\
Social function & $54.5 \pm 22.7$ & $56.5 \pm 22.2$ & $2 \pm 17.7$ & $51 \pm 22.2$ & $49.2 \pm 18.3$ \\
Role emotional & $27.4 \pm 36.7$ & $59 \pm 43.5$ & $31.5 \pm 44.2^{\mathrm{c}, \mathrm{f}}$ & $17.7 \pm 33.9$ & $19.8 \pm 39.1$ \\
Mental health & $56.7 \pm 14.6$ & $58.8 \pm 15.1$ & $2 \pm 12.2^{\mathrm{e}}$ & $51.4 \pm 10.1$ & $53.5 \pm 10.2$ \\
Mental component summary & $46.3 \pm 13.4$ & $55.9 \pm 16.9$ & $9.5 \pm 13^{\mathrm{c}, \mathrm{f}}$ & $43.7 \pm 8.5$ & $42,6 \pm 12$ \\
BDI & $14.9 \pm 9.3$ & $10.7 \pm 8.2$ & $-4.2 \pm 5.2^{\mathrm{c}}$ & $13.6 \pm 10$ & $13.1 \pm 9.9$ \\
\hline & & & & & \\
\hline
\end{tabular}

\section{Hosted file}

figure-1 - 2.docx available at https://authorea.com/users/396705/articles/509813-effectsof-supervised-versus-unsupervised-aerobic-exercise-training-on-weight-loss-functionalcapacity-quality-of-life-and-depression-level-in-patients-with-essential-hypertension 